Research Article

\title{
Effects of Porosity of Dry and Saturated Sandstone on the Energy Dissipation of Stress Wave
}

\author{
Yexue $\mathrm{Li} \mathbb{D}^{1,2}$ and Jing Wang $\mathbb{D}^{1}$ \\ ${ }^{1}$ School of Civil Engineering and Architecture, Hubei University of Arts and Science, Xiangyang, Hubei Province 441053, China \\ ${ }^{2}$ Hubei Key Laboratory of Low Dimensional Optoelectronic Material and Devices, Xiangyang, Hubei Province 441053, China \\ Correspondence should be addressed to Jing Wang; wangjing821206@163.com
}

Received 20 November 2018; Revised 22 March 2019; Accepted 7 April 2019; Published 2 May 2019

Academic Editor: Constantin Chalioris

Copyright (c) 2019 Yexue Li and Jing Wang. This is an open access article distributed under the Creative Commons Attribution License, which permits unrestricted use, distribution, and reproduction in any medium, provided the original work is properly cited.

\begin{abstract}
A SHPB experiment of porous sandstone was conducted to discuss the influencing principle of porosity on the energy dissipation of stress wave across dry and saturated porous sandstones. Changes in porosity before and after the SHPB experiment were analyzed by nuclear magnetic resonance. Results show that the number of wave peaks in the $T_{2}$ spectrum before and after the impact test of sandstone is unchanged, but the wave peak value corresponding to small pores increases. This result indicates that the sandstone structure remains in the microcrack formation stage because the stress wave energy is adequate only for forming new microcracks but not for tearing pores and increasing pore diameter. Results further show that, at the same porosity, saturated sandstone consumes less energy than dry sandstone. With increased porosity, the energy dissipation of saturated sandstone decreases, whereas that of dry sandstone increases. This phenomenon can be explained based on three aspects. First, according to the fracture mechanics theory, the surface tension of water and Stefan effect significantly hinder crack expansion. Therefore, generating a new surface to dissipate stress wave energy of saturated sandstone is more difficult than that of dry sandstone under the same porosity. Second, during impact loading, saturated sandstone is in a nondrainage state, and its deformation can be viewed approximately as the sum of compressive deformation of sandstone and water. Water has smaller comprehensive deformation than sandstone. The deformation of saturated sandstone decreases with increased porosity, i.e., water content. Plastic deformation and the probability of new cracks decrease, so energy dissipation of saturated sandstone decreases. Third, with increased porosity, the dynamic strength of dry sandstone declines accordingly. Developing new cracks during loading to dissipate stress wave energy as surface energy is easy. Therefore, high porosity causes great energy dissipation.
\end{abstract}

\section{Introduction}

Blasting is still a usual method of mineral exploration and excavation of underground chamber. After explosive is detonated, energy carried by the impact wave or stress wave propagates from the central to the outside. When stress wave encounters the discontinuous structural surfaces, such as cracks, pores, and joints, complicate transmission and reflection occur. And stress wave energy dissipates [1]. A large amount of energy for rock crushing or mining is wasted by discontinuous structural surfaces. In order to sufficiently utilize the explosion energy and enhance the efficiency of rock breaking, except for selecting appropriate explosive amount and location to install the explosive, one key scientific problem that needs to be solved is to deeply study the propagation principle and energy dissipation mechanism of the stress wave in the rocks with discontinuous structural surfaces, such as cracks, pores, and joints.

Rocks are porous media formed by aeon diagenesis. Differences in diagenesis and the surrounding environment will cause great differences in the pore structures of rocks [2]. The size and spatial location of pores in rocks are almost in random distribution. Deducing the influencing principle of microscopic and macroscopic discontinuous structural surfaces in rocks (e.g., pores, fissures, and joints) on the propagation of stress wave and explaining the internal 
influencing mechanism theoretically are difficult. Some studies neglected some secondary factors or focused on influences of some parameters. Theoretically, the effects of pores and joints on the stress wave propagation can be deduced [3-6]. The transmission and reflection coefficients of the stress wave after passing through a single joint were deduced with considerations to the effect of rough joint surface, based on fractal theory [7]. The analytical solutions for the transmission and reflection coefficients were established by the displacement discontinuity model, and the energy dissipation principle of the system was discussed $[8,9]$. However, these analytical solutions for the transmission and reflection coefficients and the energy dissipation principle of porous rocks under dynamic load are based on multiple idealizations of the model.

The energy variation principle of natural porous rocks under dynamic loads was discussed. Existing studies analyzed the variation principles of related parameters after stress wave runs through porous rocks using ANSYS, Flac3D, and finite element computing software and disclosed relations between parameters (wave velocity and energy dissipation) and rock porous parameters (location distribution of pores and porosity) $[10,11]$.

Given the complicated internal structure of rocks, rocks embody homogeneous and anisotropic properties. Complicated homogeneity and anisotropy make setting accurate initial parameters in numerical calculation difficult for researchers. Even the set initial parameters may have a great gap with the actual ones. Additionally, due to the complicated properties of rocks, perhaps an inappropriate constitutive relationship and boundary conditions are selected in the numerical calculation. Thus, the calculated results have some differences compared with the actual values.

To ensure the feasibility and engineering applicability of research results, many experiments have been conducted in existing studies to explain the response process and mechanism of rock pores under impact loading conditions. From the view of energy, the SHPB test on the specimen with two manufactured rough parallel joints was performed to study the influence of roughness on stress wave energy dissipation [12]. Two joints embedded in the rock were the idealized model of natural pore structures. Consequently relation between joint parameters and transmitted and reflected coefficients of the stress wave across the rock with many parallel joints was discussed [13]. In fact, the joints in rock mass were not parallel. Their distribution is basically random, and their directions were undetermined. Thus, an artificial porous rock with determined porosity was manufactured using similar materials to model natural rock. Then a SHPB experiment was conducted to discuss the energy dissipation principle in porous rock [14]. However, the porosity of prefabricated pores has great errors. The error in this study might be greater because of overlapping of manufacturing error and other experimental errors.

In order to reduce the error, 3D printing technology is applied to replicate the pore structure of natural volcanic rock. And its fractured process is investigated under the dynamic load [15]. Although replication of pore structure using 3D printing is especially similar to that of the natural rock, there exists difference between manufactured rock and natural rock. Subsequently, the deformation mechanism of natural rock pore under the stress wave was discussed by CT and SHPB [16]. The CT experiment can test porosity but not the diameter of pores. Thus, rocks with the same porosity but different diameters show different influencing principles on stress wave propagation tested by using SHPB. Obviously, the result is acceptable.

Nuclear magnetic resonance (NMR) technology is proposed because of the development of microstructure test technology. This technology remarkably solves the quantitative description problems of pore diameter in materials and porosity with different pore diameters [17, 18]. This technology offsets the shortcomings of CT technology. Some studies have used NMR technology to describe porous structure, effects of porous structure on mechanical properties of materials, and disclosed the damage evolution process of porous rocks under static load [19].

In general, the study object develops from one joint or many joints to pore structure made by $3 \mathrm{D}$ printing. By theoretical derivation, experimental test, and numerical analysis, fruitful investigation results on the response of pore structure under the dynamic load were obtained. However, whether one joint or pore structure made by $3 \mathrm{D}$ printing, their pore structures were still simple or simplified compared with complex pore structures of natural rock mass, so conclusion from the simplified manufactured specimen was approximate and inaccurate.

Thus, in this paper, natural sandstone was selected. NMR technology was applied to test porosity and pore diameters in rocks. The sandstones including pores with basically similar shape and diameter but different number were selected. The deformation and failure of pores under impact loading was explored. The influencing principle of changing rock porosity on stress wave propagation, and energy dissipation under dry and saturated conditions was discussed. The energy dissipation principle and the mechanism of porous sandstone were disclosed.

\section{Experimental Process}

2.1. Processing of Porous Sandstone. Porous sandstone was selected as the research object. To avoid the effects of anisotropy on the experimental results, rock masses were drilled from the same direction by using a standard driller to take the $50 \mathrm{~mm}$ (diameter) $\times 75 \mathrm{~mm}$ (length) rock core. Considering the high requirements on the accuracy of parallelism between the faces of the upper and lower ends $(\leq 0.1 \mathrm{~mm})$ and the face flatness $(\leq 0.05)$ of rocks in the impact dynamics test, a double-edge grinder was adopted to semifinish the rock samples in the experiment. In the first processing stage, semifinished rock samples were rapidly grinded to $66 \mathrm{~mm}$ using a low-accuracy double-side miller, leaving $1 \mathrm{~mm}$ for second finishing. Next, the grinder with $0.01 \mathrm{~mm}$ accuracy was used for further polishing until a preset length, and other test accuracy requirements were achieved. A total of 102 finished rock samples with $65 \mathrm{~mm}$ length and $50 \mathrm{~mm}$ diameter were formed. 
2.2. Scanning Test of Porous Sandstone. A low-field intensity NMR analyzer from Hubei Key Laboratories of Low Dimensional Optoelectronic Material and Devices was used in this experiment (Figure 1). This analyzer was mainly composed of a permanent magnet, sample cell, RF system, and data acquisition and analysis system. The magnetic field intensity of the permanent magnet was $0.5 \mathrm{~T}$ (Tesla). To maintain the uniformity and stability of the main field, the magnet temperature was retained at $32^{\circ} \mathrm{C}$ with an accuracy of $+0.01^{\circ} \mathrm{C}$. The effective test region of the sample cell was $80 \mathrm{~mm} \times \Phi 80 \mathrm{~mm}$.

All the processed rock samples were placed in a saturation box and pressurized to $0.1 \mathrm{MPa}$. These rock samples were fully saturated for $24 \mathrm{~h}$ until a constant weight was reached. Then, the scanning test of porous sandstone was performed using the low-field NMR analyzer. The detailed process was as follows: the NMR analyzer was started. Several minutes were left for smooth running. After all scanning parameters were set, a specimen was placed in the testing box of NMR, and scanning experiment was conducted to collect the transverse relaxation time $T_{2}$ spectrum. After the scanning experiment was finished, all $T_{2}$ spectra were inverted into rock pore diameter intervals and pore volume curves. Pore diameter and porosity data were stored in Excel files for later processing. The aperture type of different rock samples in the SHPB test must be uniform because this study discusses the influences of the porosity of dry and saturated sandstones on stress wave propagation principle. Generally, different sandstones have diverse porous structures. Therefore, many NMR experiments of sandstone must be implemented to screen one group of rock samples with the similar shape and diameter of the aperture for impact loading experiment. Finally, the porous internal structure of 102 sandstone samples was tested. A total of 18 rock samples with similar pore diameter interval and shapes were selected. This selection avoided maximum extent of the negative effects of the differences in pore size on the research results. After the SHPB test, all sandstone samples were saturated again. The internal porous structural characteristics of sandstone after the impact were tested using the NMR analyzer. The variations of the porosity of dry and saturated sandstone before and after the impact tests were analyzed.

2.3. SHPB Test of Porous Sandstone. In this experiment, the SHPB device was applied in the material impact dynamics laboratory. This device is mainly composed of a nitrogen cylinder that stores high-pressure gas, bullet for impact loading, laser speedometer to measure the bullet impact velocity, incident and transmission bars as the stress wave propagation media, energy absorption bar, and device protective damper. Cylinder steel bars with a diameter of $50 \mathrm{~mm}$ and lengths of $3.5 \mathrm{~m}$ and $2 \mathrm{~m}$ were used as incident and transmission bars, respectively. The bullet was $800 \mathrm{~mm}$ in length and $50 \mathrm{~mm}$ in diameter. Resistance strain gauges were pasted on the incident and transmission bars at $2250 \mathrm{~mm}$ and $670 \mathrm{~mm}$ away from the bar end, respectively (Figure 2).

This study discusses the effects of sandstone pores on stress wave propagation and energy dissipation. The impact speed in this experiment should not be too high because over

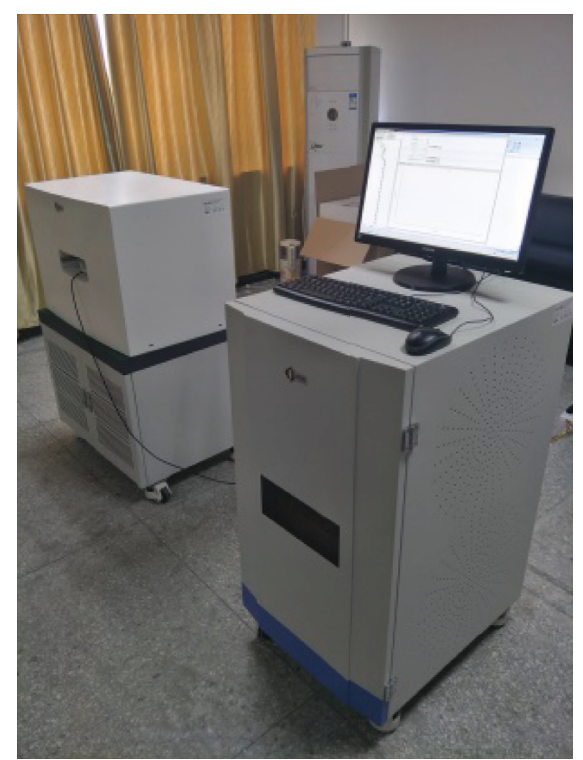

FIGURE 1: NMR analyzer.

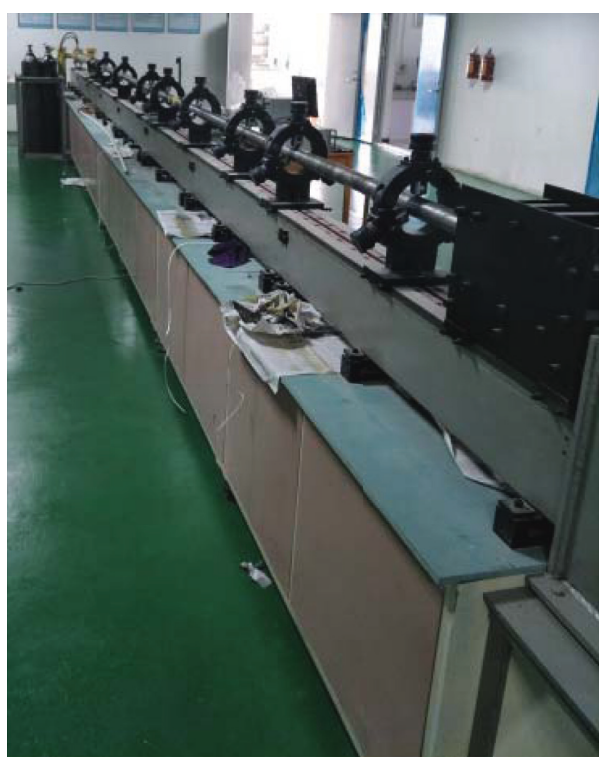

Figure 2: SHPB devices.

high impact speed might cause visible microcracks and even cracking of rock samples. This requirement does not conform to the experimental goal. Similarly, very small impact speed and initial energy are inapplicable because very small input energy will amplify occasional error in the experimental process and even cause failure in the experiment. Therefore, the bullet impact speed was adjusted by changing input air pressure in the bore of a gun before the formal experiment. After repeated experiment, $0.45 \mathrm{~atm}$ was selected as the input air pressure and approximately $8 \mathrm{~m} / \mathrm{s}$ was selected as the bullet impact speed.

After the parameters were set, a specimen was installed between the ends of the incident and transmitted rebar. Meanwhile, highly compressed nitrogen was pumped into the gun's chamber till air pressure reached $0.45 \mathrm{~atm}$. the 
trigger button was pressed, and highly compressed nitrogen impacted the incident rebar to engender the stress wave. Stress wave propagated along the incident rebar. Stress wave transmitted and reflected when it encountered the rock with discontinuous media. Finally the incident, reflected, and transmitted waves were recorded by strain gauge.

\section{Structural Analysis of Porous Sandstone}

3.1. Internal Structural Characteristics of Porous Sandstone. To discuss the internal porosity characteristics of sandstone and the effects of porosity on energy dissipation of stress wave, the four-peak $T_{2}$ spectra of sandstone were also given for comparative analysis, except for three-peak $T_{2}$ spectra of 18 sandstone samples in $\mathrm{SHPB}$. The $T_{2}$ spectra of transverse relaxation are shown in Figure 3. In Figure 3, the fourth wave peak seemed not be shown. It is because that the ratio of large pore with diameter greater than $43.2 \mathrm{~mm}$ is very small (Figure 3 and Table 1).

In all $T_{2}$ spectra, the relaxation spectra (Figure 3) with the same number of peaks were analyzed. Despite of some differences in the starting, vertex, and end times of the peak, the corresponding relaxation time of every peak from left to right is approximately in the same time interval, horizontal span between two adjacent troughs, indicating that different sandstone samples in the same batch of sandstone have basically identical pore diameter and type. In addition, in all spectra, wave peaks with basically similar horizontal coordinate interval were different. Especially the first wave peaks characterizing the pore with small diameter were distinct. It was indicated that the volumes of pores with basically similar diameter in different sandstone were various, or sandstone porosities were different. This was also the demand that the rock sample should satisfy the SHPB test, i.e., rock pores with basically similar shape and diameter but distinct number and volume.

The aforementioned analysis is qualitative. The areas surrounded by peaks and horizontal coordinates were calculated to further disclose the internal structure characteristics of sandstone. The areas were referred to the pore volumes. The volumes divided by rock total volumes obtained porosities (Table 2). Subsequently, the pore size and distribution of different types of sandstone were calculated and analyzed (Table 1).

According to the NMR principle, the transverse relaxation time is determined by the free, surface, and diffuse relaxation times:

$$
\frac{1}{T_{2}}=\frac{1}{T_{2 \text { free }}}+\frac{1}{T_{2 \text { surface }}}+\frac{1}{T_{2 \text { diffuse }}} .
$$

In this experiment, water-saturated sandstone samples were used. Its surface relaxation time is approximately $3 \mathrm{~s}$, which is far longer than the transverse relaxation time $\left(T_{2}\right)$. Besides, the diffuse relaxation time is significantly longer than $T_{2}$. The effects of the two latter items in equation (1) are neglected because the surface relaxation time and diffuse relaxation time has a gap of several magnitude levels with $T_{2}$; thus, it can be simplified as:

$$
\frac{1}{T_{2}}=\frac{1}{T_{2 \text { free }}}=\rho\left(\frac{S}{V}\right)=F_{s}\left(\frac{\rho}{r}\right) .
$$

The free relaxation time is determined by the specific surface area of materials. Pores are supposed as ideal cylinders. Thus, the geometric shape factor of pores $\left(F_{s}\right)$ is 2 . The transverse relaxation rate $(\rho)$ is $10 \mu \mathrm{m} / \mathrm{ms}$. The relationship between relaxation time and pore diameter can be expressed as

$$
r=20 T_{2}
$$

where $r$ is the pore diameter.

Equation (3) shows that the pore diameter is approximately proportional to $T_{2}$. The pore diameter can be obtained by multiplying $T_{2}$ by 20 . The corresponding proportion and porosity of pore are calculated by the sandstone volume and the area surrounded by $T_{2}$ spectral curves and $x$-axis.

The relaxation time on $T_{2}$ spectra discloses the information about the size of pores in materials. Long relaxation time reflects large pore diameter. The area formed by $T_{2}$ spectral curves and $x$-axis discloses the pore volume in sandstones. The area and pore volume are approximately proportional. Changes of the area below the curves reflect the changes of pore volumes in sandstone. Figure 4 and Table 1 show that area of the smallest pores $(0.0002 \sim 0.08 \mathrm{~mm})$, that is, the first wave peak from the left, is approximately 7531 and its pore volume ratio is $80 \%$. When the pore diameter increases to $0.08 \mathrm{~mm}-1.72 \mathrm{~mm}$, then the area formed by the spectral peaks decreases to 1529 , and the pore volume ratio is $16 \%$. As the pore diameter increases to $2 \mathrm{~mm}-15 \mathrm{~mm}$, pores have been developed into visible fissures and the corresponding spectral peak area is only 351 . The pore volume ratio is $4 \%$. In other words, on $T_{2}$ spectra, high pore diameter or long relaxation time corresponds to small area and low pore volume. This analysis is also proven by the four-peak pie graph, which shows that pores with small diameter have great proportion. In other words, sandstone contains relatively more small pores but relatively fewer large pores.

\subsection{Comparison of Pore Characteristics before and after} Impacts. The NMR relaxation spectra of sandstones (Figure 5), for 92\# dry sandstone and 64\# saturated sandstone show that the relaxation spectra of the same sample after the impact experiment still have three peaks, but the value of small pore peaks increased to some extent. This result demonstrates that the new pores with small pore diameter are developed during the impact process but existing small pores are not torn, concentrated, and finally connected into large ones due to tensile stress. This is because small impact speed and input energy were used in this SHPB experiment. Energy size can only form new small pores but is inadequate to make sample deformation enter the second state of impact loading failure, i.e., to make small pores concentrate and connect into large ones. As a result, NMR and SHPB experimental results can be verified mutually. 


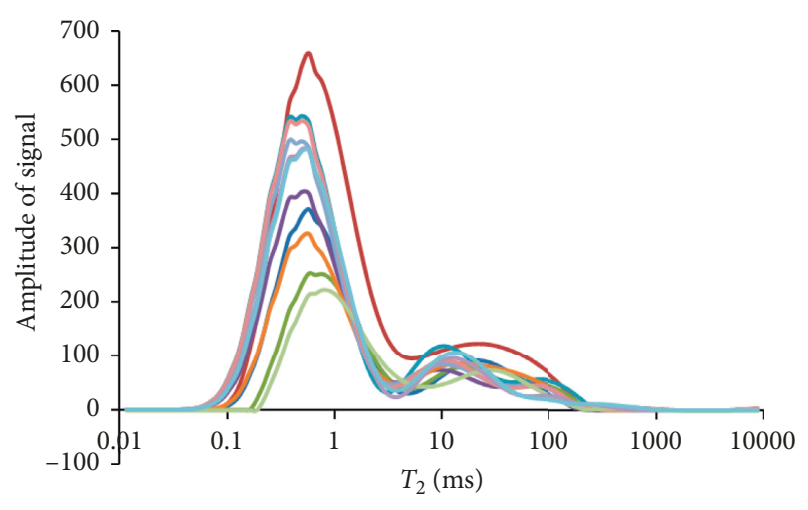

(a)

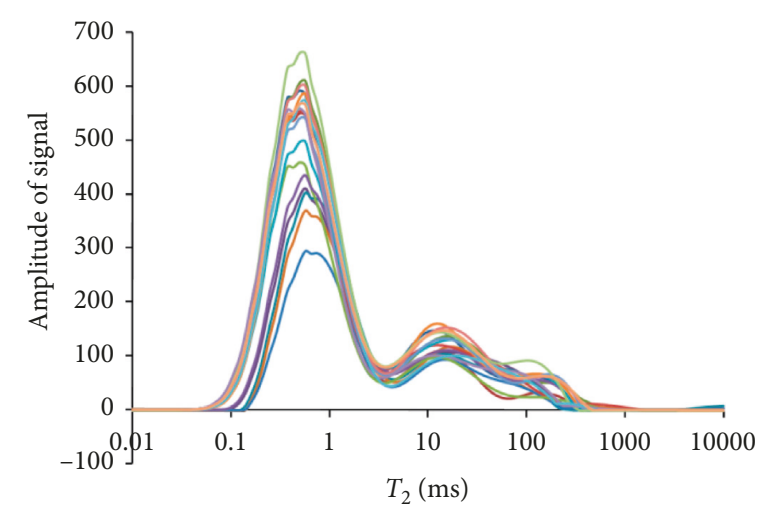

(b)

Figure 3: $T_{2}$ spectra of sandstone. (a) Three-peak spectra. (b) Four-peak spectra.

TABLE 1: Sandstone pore parameters.

\begin{tabular}{|c|c|c|c|c|c|}
\hline Type of spectra & No. of peak & Starting time & Vertex time & End time & Pore diameter range $(\mathrm{mm})$ \\
\hline \multirow{3}{*}{ Three peak } & 1 & 0.01 & 0.5 & 4 & $0.0002-0.08$ \\
\hline & 2 & 4 & 14 & 86 & $0.08-1.72$ \\
\hline & 3 & 100 & 150 & 750 & $2-15$ \\
\hline \multirow{4}{*}{ Four peak } & 1 & 0.01 & 0.5 & 5 & $0.0002-0.1$ \\
\hline & 2 & 8 & 28 & 240 & $0.16-4.8$ \\
\hline & 3 & 265 & 403 & 2154 & $5.3-43.08$ \\
\hline & 4 & 2160 & 3000 & - & $>43.2$ \\
\hline
\end{tabular}

TABLE 2: Statistics on porosity and energy.

\begin{tabular}{|c|c|c|c|c|c|}
\hline & No. & Porosity (\%) & $\begin{array}{l}\text { Reflected wave energy } \\
\text { ratio }\left(W_{\mathrm{r}} / W_{\mathrm{i}}\right)(\%)\end{array}$ & $\begin{array}{l}\text { Transmitted wave energy } \\
\text { ratio }\left(W_{\mathrm{t}} / W_{\mathrm{i}}\right)(\%)\end{array}$ & $\begin{array}{l}\text { Energy dissipation } \\
\text { ratio }\left(W_{\mathrm{d}} / W_{\mathrm{i}}\right)(\%) \\
\end{array}$ \\
\hline \multirow{9}{*}{ Dry sandstone } & 70 & 4.59 & 60.54 & 18.22 & 21.24 \\
\hline & 92 & 5.59 & 59.34 & 14.42 & 26.24 \\
\hline & 86 & 6.13 & 49.04 & 19.63 & 31.33 \\
\hline & 80 & 6.26 & 47.71 & 27.32 & 24.97 \\
\hline & 24 & 6.67 & 72.17 & 9.21 & 18.63 \\
\hline & 95 & 7.51 & 56.41 & 14.41 & 29.18 \\
\hline & 67 & 8.36 & 64.75 & 11.64 & 23.61 \\
\hline & 98 & 8.57 & 60.16 & 14.68 & 25.16 \\
\hline & 94 & 9.30 & 58.27 & 13.65 & 28.08 \\
\hline \multirow{9}{*}{ Saturated sandstone } & 64 & 6.57 & 78.25 & 6.62 & 15.13 \\
\hline & 61 & 7.72 & 76.03 & 8.87 & 15.10 \\
\hline & 39 & 7.97 & 73.81 & 8.03 & 18.17 \\
\hline & 53 & 8.18 & 62.43 & 21.16 & 16.41 \\
\hline & 59 & 8.32 & 81.70 & 5.30 & 13.00 \\
\hline & 60 & 8.49 & 74.01 & 10.31 & 15.69 \\
\hline & 36 & 8.66 & 80.45 & 6.10 & 13.45 \\
\hline & 55 & 8.90 & 77.07 & 8.22 & 14.72 \\
\hline & 54 & 9.58 & 77.32 & 10.09 & 12.59 \\
\hline
\end{tabular}

\section{Analysis of Stress Wave Energy Features in Porous Sandstone}

4.1. Qualitative Analysis of the Effects of Porosity on Energy Transmission. To discuss the influence principle of porosity on stress wave energy transmission, a comparison analysis between the energy propagation characteristics of sandstone and marble (previous research results) during impact loading was conducted. The stress time-history curves of two sandstone samples are shown in Figure 6. The reflected wave peak is large and is slightly smaller than the incident wave peak. In contrast, the transmitted wave peak is smaller. In other words, a large proportion of energy, which is carried by the stress wave, is reflected by the porous sandstone, and 


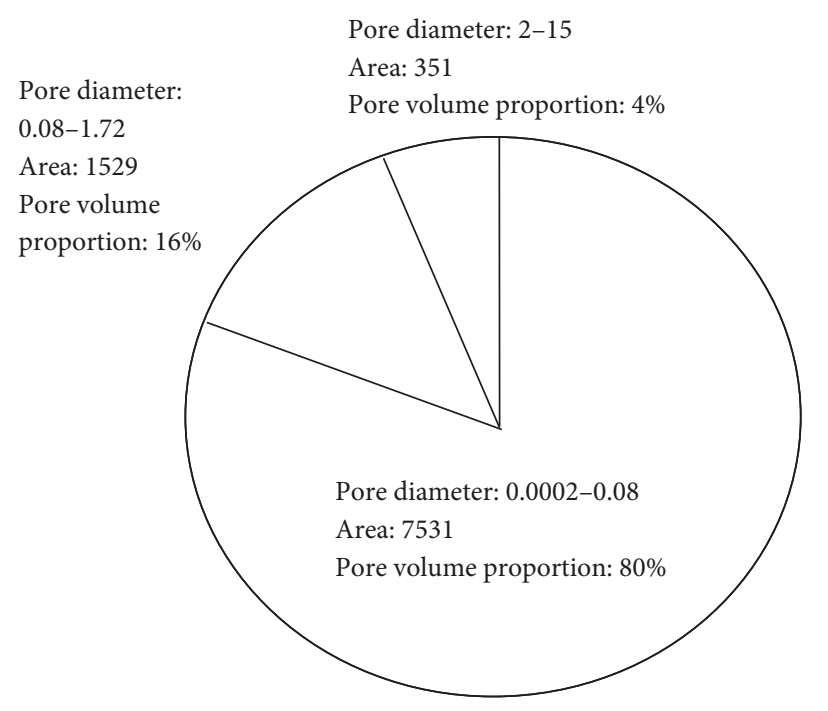

(a)

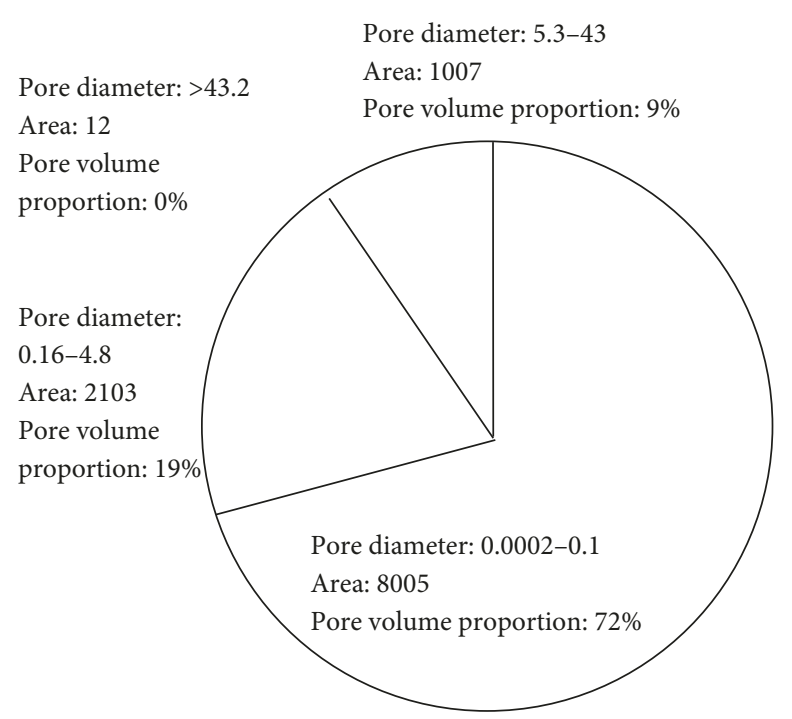

(b)

Figure 4: Pie graphs of pore diameter and volume. (a) Three peak. (b) Four peak.

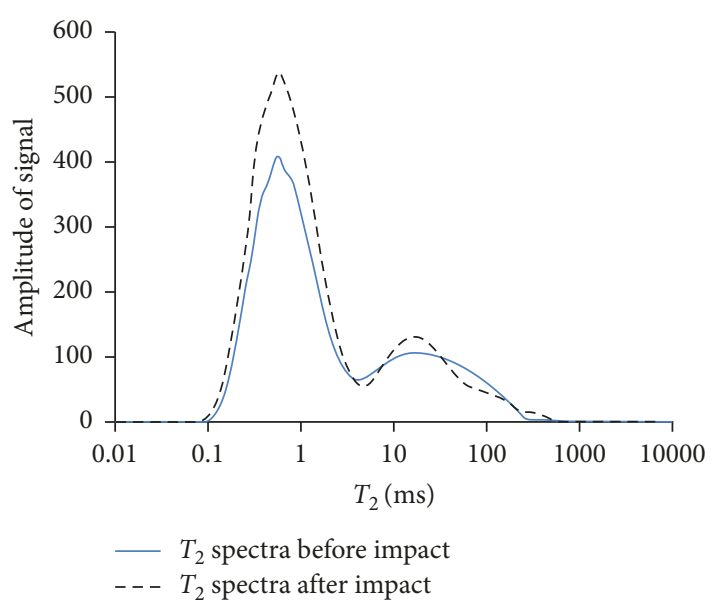

(a)

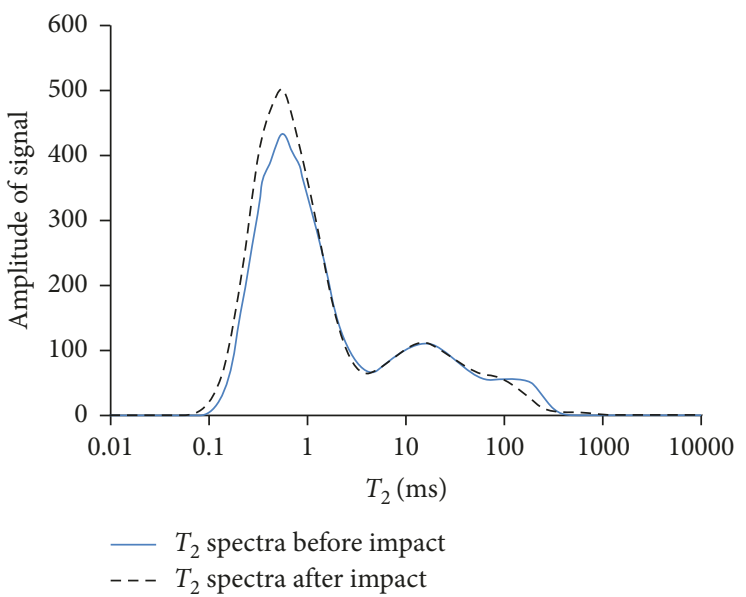

(b)

FiguRE 5: Comparison of $T_{2}$ spectra of sandstone samples before and after SHPB experiment. (a) 92\# dry sandstone. (b) 64\# saturated sandstone.

only a small proportion of energy can pass through the porous sandstone. However, the experimental results of marble show otherwise. A small proportion of energy is reflected, and a large proportion of energy is transmitted.

What is cause or mechanism of the experimental phenomenon? The experimental results in the NMR of rocks show that the porosities of marble and sandstone are $0.1 \%$ and $6 \%$, respectively. When the stress wave runs through porous rocks, stress acts on solid media and the media-pore interface, respectively. The function of solid media is to carry and transmit stress waves, whereas the function of mediapore interface is mainly to reflect stress waves. With increased porosity, the proportion of solid media for transmitting stress wave decreases, whereas the proportion of interface for reflecting stress wave increases. Thus, when the stress wave runs through sandstones with large porosity, the transmission energy is small and the reflection energy is large. When the stress wave runs through marble with small porosity, the transmission energy is large and the reflection energy is small.

4.2. Quantitative Analysis of Effects of Porosity on Stress Wave Energy. According to the one-dimensional stress assumption of SHPB, the energy equations of incident, reflected, and transmitted waves can be expressed uniformly as

$$
W=\frac{A}{C \rho} \int_{0}^{T} \sigma^{2}(t) d t
$$

where $W$ is the stress wave energy, $\rho$ is the volume density of steel bar, $C$ is the velocity of stress wave propagating in the bar, $A$ is the cross-sectional area of the bar, $T$ is the wave 

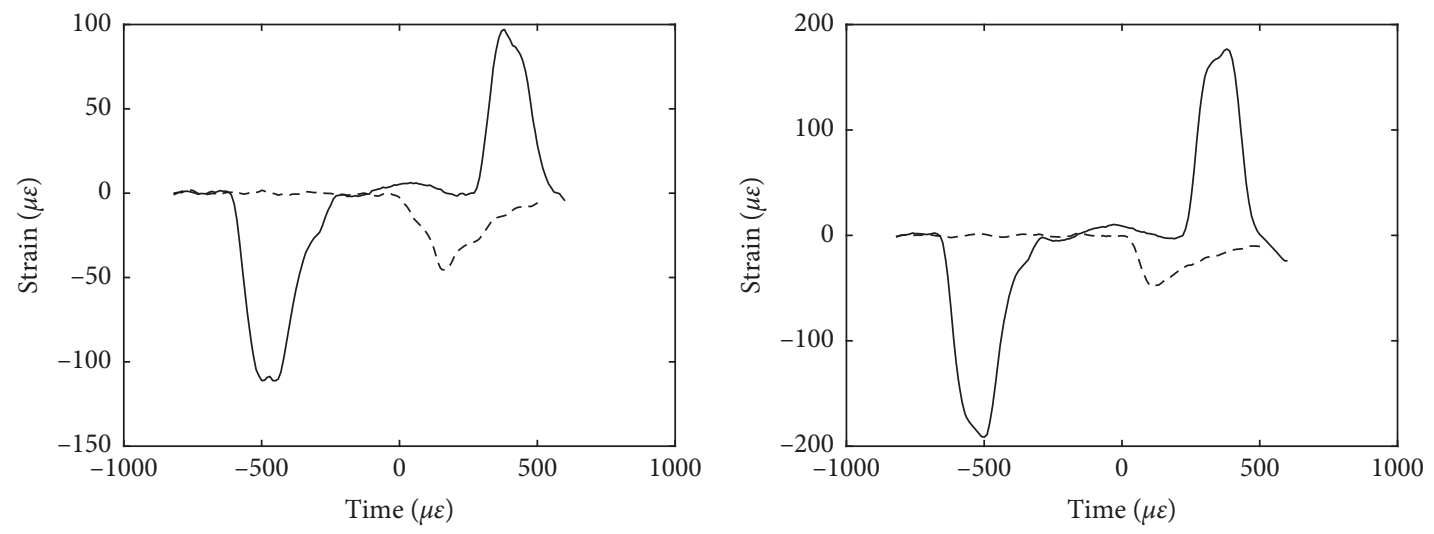

_ Incident wave, reflected wave - - - Transmitted wave

— Incident wave, reflected wave - - - Transmitted wave

(a)

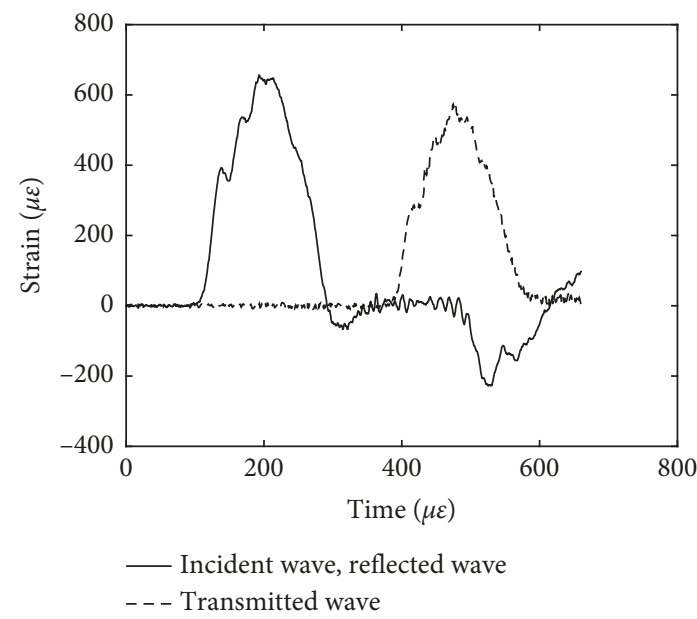

(c)

(b)

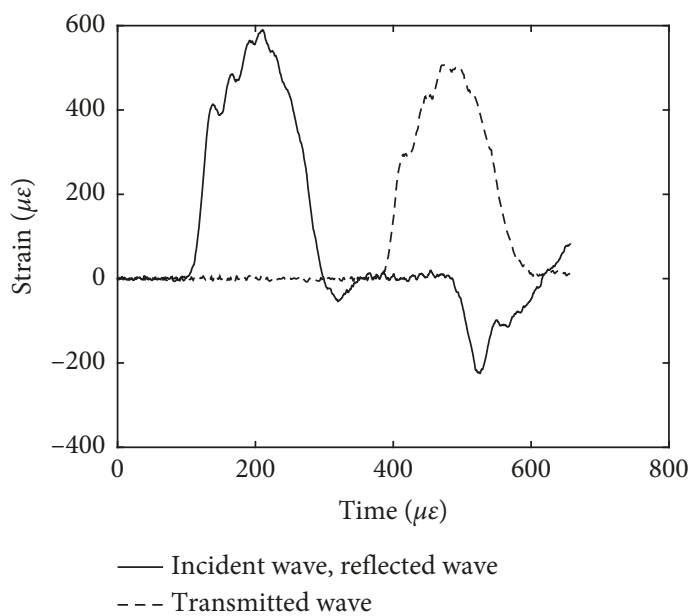

(d)

FiguRE 6: Stress waveform of marble and sandstone. (a) 92\# dry sandstone. (b) 64\# saturated sandstone. (c) Marble 1 (previous research results). (d) Marble 2 (previous research results).

propagation time, and $\sigma(t)$ is the stress wave propagated in the bar. The corresponding stress wave expression is brought in and the energy equation set can be obtained:

$$
\left\{\begin{array}{l}
W_{i}=\frac{A}{C \rho} \int_{0}^{T} \sigma_{\mathrm{i}}^{2}(t) d t \\
W_{r}=\frac{A}{C \rho} \int_{0}^{T} \sigma_{\mathrm{r}}^{2}(t) d t \\
W_{t}=\frac{A}{C \rho} \int_{0}^{T} \sigma_{\mathrm{t}}^{2}(t) d t
\end{array}\right.
$$

According to the energy conservation law,

$$
W_{\mathrm{i}}=W_{\mathrm{d}}+W_{\mathrm{r}}+W_{\mathrm{t}} .
$$

Bring (5) into (6), we obtain

$$
W_{\mathrm{d}}=\frac{A}{C \rho}\left[\int_{0}^{T} \sigma_{\mathrm{i}}^{2}(t) d t_{1}-\int_{0}^{T} \sigma_{\mathrm{r}}^{2}(t) d t-\int_{0}^{T} \sigma_{\mathrm{t}}^{2}(t) d t\right],
$$

where $W_{\mathrm{d}}$ is the energy dissipation and $\sigma_{\mathrm{i}}(t), \sigma_{\mathrm{r}}(t)$, and $\sigma_{\mathrm{t}}(t)$ are incident, reflected, and transmitted waves, respectively. According to (5) and (7), the computing programs of incident, reflected, and transmitted wave energies and energy dissipation were compiled by using MATLAB2015. Stress wave data of 18 sandstone samples were input into the programs, thus calculating the energy value and dissipation of the stress wave. Because the incident wave energy in each loading process is not identical, all data were normalized to ensure the comparability of research conclusions. The reflected and transmitted wave energies and the dissipated energy of dry sandstone and saturated sandstone are divided by their incident wave energy. Three new parameters, namely, reflected wave energy ratio $\left(W_{\mathrm{r}} / \mathrm{W}_{\mathrm{i}}\right)$, transmitted wave energy ratio $\left(W_{\mathrm{r}} / W_{\mathrm{i}}\right)$, and energy dissipation ratio $\left(W_{\mathrm{r}} /\right.$ $W_{\mathrm{i}}$ ), were introduced to discuss the influences of porosity on these three parameters under dry and wet saturation conditions. The calculated results are shown in Table 2 . In Figures 7 and 8, the triangle point and prismatic point represent the related data of dry sandstone and saturated 


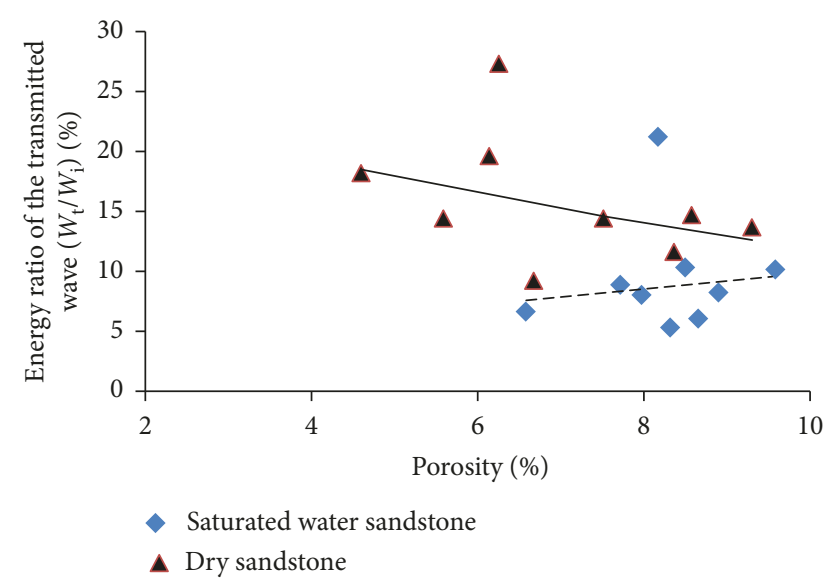

FIGURE 7: Effect of porosity on transmitted wave energy.

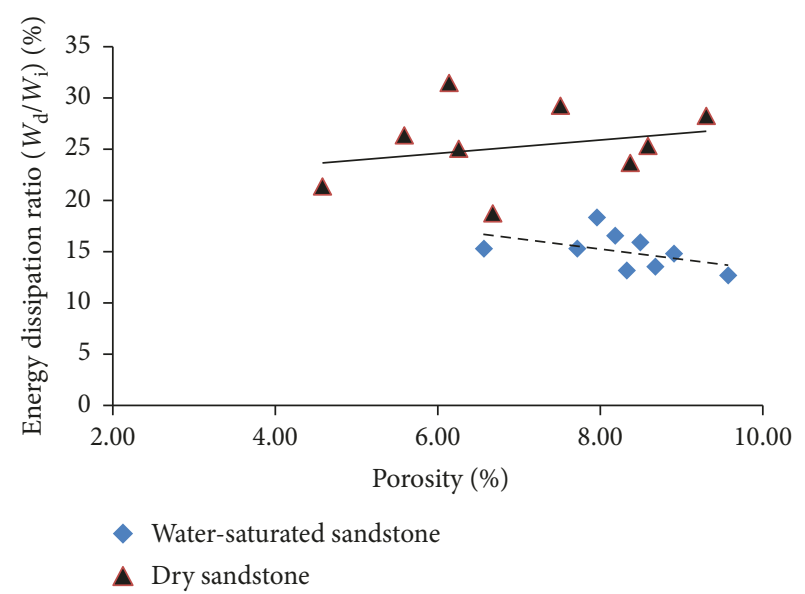

FIGURE 8: Relationship between porosity and energy dissipation ratio.

sandstone, and the solid line and dotted line represent the trend line of related data of dry sandstone and of saturated sandstone, respectively.

4.2.1. Effects of Porosity on Transmitted Wave Energy. According to the impact dynamics experimental results of dry porous sandstone (Figure 7 ), $W_{\mathrm{t}} / W_{\mathrm{i}}$ fluctuates with the porosity in a certain range when the stress wave runs through dry sandstone. Their relation curve shows that the relationship between $W_{\mathrm{t}} / W_{\mathrm{i}}$ and porosity is clear. The transmitted energy of the stress wave decreases with increased the porosity. SHPB experimental results of saturated sandstone reflect the opposite; that is, the transmitted energy of stress wave increases with increased the porosity of saturated sandstone.

4.2.2. Effects of Porosity on Reflected Wave Energy. Figure 7 and Table 2 show that approximately 60\% energies are reflected back to the incident bar when the stress wave runs through the dry sandstone, and only approximately $15 \%$ energy runs through the sandstone and enters into the transmission bar. For saturated sandstone, approximately
$77 \%$ energies are reflected by the stress wave, and only approximately $8 \%$ energies are transmitted by rocks. That is, for dry and saturated sandstones, most energies of the stress wave are reflected in the incident bar but only a small proportion of energy runs through rock samples. Differently, the saturated sandstone has higher proportion of reflected energy and smaller proportion of transmitted energy than dry sandstone with the same porosity.

4.2.3. Energy Dissipation Analysis of Stress Wave. In the impact dynamic test, many forms of energy dissipation, such as plastic deformation energy due to plastic deformation of sandstone, kinetic energy that sandstone flies off after impact, surface energy dissipated through new surface formed by crack generation and expansion, acoustic emission energy accompanied in sandstone breakage, and internal energy, were found. Existing studies have demonstrated that acoustic emission energy and internal energy account for less than $5 \%$ of the total dissipated energy; thus, they can be neglected. Furthermore, input energy is relatively small during the SHPB test; hence, the sandstone samples will not fly off, and no kinetic energy was observed. Therefore, plastic deformation energy and surface energy are the main causes of energy dissipation in the test. The effects of these two kinds of energy on energy dissipation are discussed in the following section. In addition, it should be noted that testing porosity by nuclear magnetic resonance is indirectly conducted by detecting the hydrogen atom in the porous water. Thus, a porosity of water-saturated rock is approximately proportional to water content, i.e., the larger the porosity, the higher the water content.

According to the relation curve between energy dissipation ratio and porosity (Figure 8), the energy dissipation of saturated sandstone is smaller than that of dry sandstone under the same porosity. The comparison analysis of the NMR test of sandstone before and after the impact experiment shows that the pore volumes of dry and saturated sandstone samples increase to some extent after the impact experiment. However, the pore volume of saturated sandstone increases slightly (Figure 5) and only few new small pores are formed, indicating that compared with dry sandstone, saturated sandstone has relatively smaller proportion of stress wave energy dissipated as surface energy during the impact process. NMR experimental results and SHPB test results can verify mutually.

Additionally, although the energy dissipation ratio fluctuates in a certain range with porosity, the fitting curve between energy dissipation ratio and porosity discloses clearly that the $W_{\mathrm{d}} / W_{\mathrm{i}}$ increases with the increased porosity of dry sandstone. However, saturated sandstone shows otherwise, that is, with increased porosity, $W_{\mathrm{d}} / W_{\mathrm{i}}$ decreases.

\subsection{Mechanism Explanation on Energy Dissipation Variation Principle}

4.3.1. Mechanism Explanation Based on Fracture Mechanics Theory. The fracture mechanics theory implies that the pore water in saturated sandstone in this experiment is free water. 
Under impact loading conditions, water has not enough time to penetrate into crack tips; thus, water still stays at the back positions of the cracks. Crack expansion is hindered significantly due to surface tension and Stefan effect of water. Resistance that is generated by surface tension is

$$
F_{1}=\frac{V \gamma}{2 \delta^{2} \cos \theta},
$$

where $V$ is the water volume, $\gamma$ is the surface energy, $\theta$ is the wetting angle, and $\delta$ is the radius of water meniscus. Resistance that is generated by Stefan effect is

$$
F_{2}=\frac{3 \pi \alpha R^{4} d u}{2 h^{3} d t},
$$

where $\alpha$ is the liquid viscosity, $R$ is the radius of the crack surface, $h$ is the distance between two surfaces of the crack, and $u$ is the relative speed for the separation of two surfaces of the crack. The resistance inhibits the expansion of cracks and the generation of new surfaces. Therefore, the "channel" for stress wave energy dissipated as surface energy in saturated sandstone narrows. Meanwhile, given that saturated sandstone is difficult to compress and is even incompressible during the impact loading process, the probability of generating plastic deformation energy reduces greatly. In a word, saturated sandstone has lower energy dissipation than dry sandstone under the same porosity due to surface tension and Stefan effect of water.

\subsubsection{Mechanism Explanation Based on Dynamic Strength} Theory of Rocks. For dry sandstone, pores will weaken the dynamic strength of rocks. Porosity is negatively related with the dynamic strength of sandstone. Plastic deformation and microcracks under dynamic loading are easier to generate with the reduction of strength of the sandstone. Besides, the probability of plastic deformation and new surface formation increases continuously with the reduction of strength. From the viewpoint of energy, plastic potential energy and surface energy increase with the increased porosity, thereby increasing the energy dissipation ratio of the stress wave.

For saturated sandstone, porosity is positively related with dynamic strength because saturated sandstone is in a nondrainage state under high-speed impact loading. The larger the porosity, the higher the water content, so dynamic strength is the higher. With increased strength, sandstone is more difficult to crack. From the viewpoint of energy, stress wave energy dissipation decreases because less new surfaces are formed.

\subsubsection{Mechanism Explanation Based on Deformation} Characteristics of Sandstone. The deformation characteristics of saturated sandstone show that it is in the nondrainage state under impact loading and the impact load is shared by sandstone and water. The deformation of saturated sandstone can be approximately considered by the sum of deformation of sandstone and water. Because the compressibility of water is lower than that of solid media in sandstone, the deformation of saturated sandstone decreases with increased porosity of saturated sandstone or water content of samples. Meanwhile, the possibility of new cracks declines. From the viewpoint of energy, the increase in porosity of saturated sandstone decreases the energy dissipation ration of the stress wave.

\section{Conclusions}

The effects of porosity on the energy dissipation of the stress wave are discussed based on NMR test and SHPB experiment of dry and saturated sandstones. The internal mechanisms of the experimental conclusions are explained. The major research conclusions are

(1) The $T_{2}$ spectra of sandstones before and after the impact loading show that the $T_{2}$ spectra of the same sandstone still have three peaks, but the value of the first peak increased to some extent. This result reflects that new cracks are developed in rocks after impact loading. However, the impact energy is inadequate to tear existing cracks into large ones because under low-speed impact loading, sandstone deformation stays in the stage of microcrack formation due to the small input energy of the stress wave. The energy is inadequate to expand cracks and tear small pores to large cracks.

(2) Given the same porosity, saturated sandstone consumes lesser energy than dry sandstone. With increased porosity, the energy dissipation of saturated sandstone decreases, whereas the energy dissipation of dry sandstone increases. This phenomenon can be explained by the fracture mechanic theory, dynamic strength theory, and rock deformation characteristics. (a) According to the fracture mechanics theory, free water in sandstone cannot penetrate into crack tips and stay at the back positions of cracks during the rapid impact loading process. The expansion of crack is significantly inhibited because of the surface tension of water and Stefan effect, thus reducing the probability of plastic deformation and formation of new surfaces. Therefore, saturated sandstone will dissipate less stress wave energy as plastic deformation energy and surface energy than dry sandstone under the same porosity. (b) Pores in dry sandstone weaken the strength of a rock. With increased porosity, the strength of porous sandstone declines continuously. During impact loading, the probability of forming new pores increases accordingly due to the breaking of solid media in sandstone. From the viewpoint of energy, energy dissipation increases with the increased porosity. (c) Saturated sandstone is in the nondrainage state during impact loading. The deformation of saturated sandstone can be approximately considered the sum of compressive deformation of solid media and water. Under fixed loads, the compressive deformation of water is smaller than that of solid media. With increased porosity, i.e., increased water content, the total deformation of porous sandstone declines. The probability of plastic deformation energy and new crack 
formation decreases. Energy dissipation also decreases correspondingly.

\section{Data Availability}

The data used to support the findings of this study are available from the corresponding author upon request.

\section{Conflicts of Interest}

The authors declare that they have no conflicts of interest.

\section{Acknowledgments}

This work had been funded by the Key project of the Hubei Provincial Department of Education (grant no. D20172601).

\section{References}

[1] Y. Potvin and J. Wesseloo, "Towards an understanding of dynamic demand on ground support," Journal of the Southern African Institute of Mining and Metallurgy, vol. 113, no. 12, pp. 913-922, 2013.

[2] W.-H. Wang, X.-B. Li, Y.-P. Zhang, and Y.-J. Zuo, "Closure behavior of rock joint under dynamic loading," Journal of Central South University of Technology, vol. 14, no. 3, pp. 408-412, 2007.

[3] J. Zhao, X. B. Zhao, and J. G. Cai, "A further study of P-wave attenuation across parallel fractures with linear deformational behaviour," International Journal of Rock Mechanics and Mining Sciences, vol. 43, no. 5, pp. 776-788, 2006.

[4] J. C. Li, X. B. Zhao, H. B. Li, S. B. Chai, and Q. H. Zhao, "Analytical study for stress wave interaction with rock joints having unequally close-open behavior," Rock Mechanics and Rock Engineering, vol. 49, no. 8, pp. 3155-3164, 2016.

[5] N. Babanouri and H. Fattahi, "Evaluating orthotropic continuum analysis of stress wave propagation through a jointed rock mass," Bulletin of Engineering Geology and the Environment, vol. 77, no. 2, pp. 725-733, 2018.

[6] J. B. Zhu, X. B. Zhao, J. C. Li, G. F. Zhao, and J. Zhao, "Normally incident wave propagation across a joint set with the virtual wave source method," Journal of Applied Geophysics, vol. 73, no. 3, pp. 283-288, 2011.

[7] Y. Li, Z. Zhu, B. Li, J. Deng, and H. Xie, "Study on the transmission and reflection of stress waves across joints," International Journal of Rock Mechanics and Mining Sciences, vol. 48, no. 3, pp. 364-371, 2011.

[8] Y. Li and Z. Zhu, "study on the velocity of P waves across a single joint based on fractal and damage theory," Engineering Geology, vol. 151, no. 29, pp. 82-88, 2012.

[9] J. B. Zhu, A. Perino, G. F. Zhao et al., "Seismic response of a single and a set of filled joints of viscoelastic deformational behaviour," Geophysical Journal International, vol. 186, no. 3, pp. 1315-1330, 2011.

[10] J. Yu, "Effects of single joint with different nonlinear normal deformational behaviors on P-wave propagation," in Geotechnical Engineering for Disaster Mitigation and Rehabilitation, pp. 458-465, Springer, Berlin, Heidelberg, Germany, 2008.

[11] J. Guan and H. Chen, "Numerical analysis of welded joint treated by explosion shock waves," Frontiers of Materials Science in China, vol. 1, no. 2, pp. 197-202, 2007.
[12] Y. Li, H. Pan, and Q. Li, "Influence of two rough parallel joint surface profiles on stress wave energy dissipation," Geotechnical Engineering, vol. 49, no. 3, pp. 47-54, 2018.

[13] X. Huang, S. Qi, S. Guo, and W. Dong, "Experimental study of ultrasonic waves propagating through a rock mass with a single joint and multiple parallel joints," Rock Mechanics and Rock Engineering, vol. 47, no. 2, pp. 549-559, 2014.

[14] X. I. A. Chang-jing, X. I. E. He-ping, and J. U. Yang, "Experimental study of energy dissipation of porous rock under impact loading," Engineering Mechanics, vol. 23, no. 9, pp. 1-5, 2006, in Chinese.

[15] J. B. Zhu, T. Zhou, Z. Y. Liao, L. Sun, X. B. Li, and R. Chen, "Replication of internal defects and investigation of mechanical and fracture behaviour of rock using $3 \mathrm{D}$ printing and 3D numerical methods in combination with X-ray computerized tomography," International Journal of Rock Mechanics and Mining Sciences, vol. 106, pp. 198-212, 2018.

[16] J. Yang, Y. M. Yang, Y. Z. Mao, H. B. Liu, and H. J. Wang, "Laboratory investigation on mechanisms of stress wave propagations in porous media," Science in China Series E: Technological Sciences, vol. 52, no. 5, pp. 1374-1389, 2009.

[17] F. Shi, C. Zhang, J. Zhang, X. Zhang, and J. Yao, "The changing pore size distribution of swelling and shrinking soil revealed by nuclear magnetic resonance relaxometry," Journal of Soils and Sediments, vol. 17, no. 1, pp. 61-69, 2017.

[18] M. A. Chesta, M. E. Ramia, S. Jeandrevin, and C. A. Martín, "Nano-pore size and porosity study by means of nuclear magnetic resonance and positronium annihilation lifetime," Applied Physics A, vol. 97, no. 2, pp. 301-307, 2009.

[19] J. Li, K. Zhou, and B. Ke, "Association analysis of pore development characteristics and uniaxial compressive strength property of granite under freezing-thawing cycles," Journal of China Coal Society, vol. 40, no. 8, pp. 1783-1789, 2015, in Chinese. 


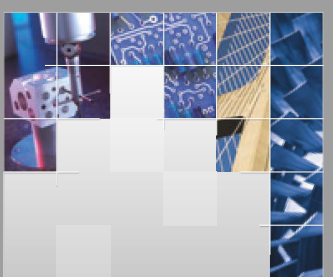

\section{Enfincering}
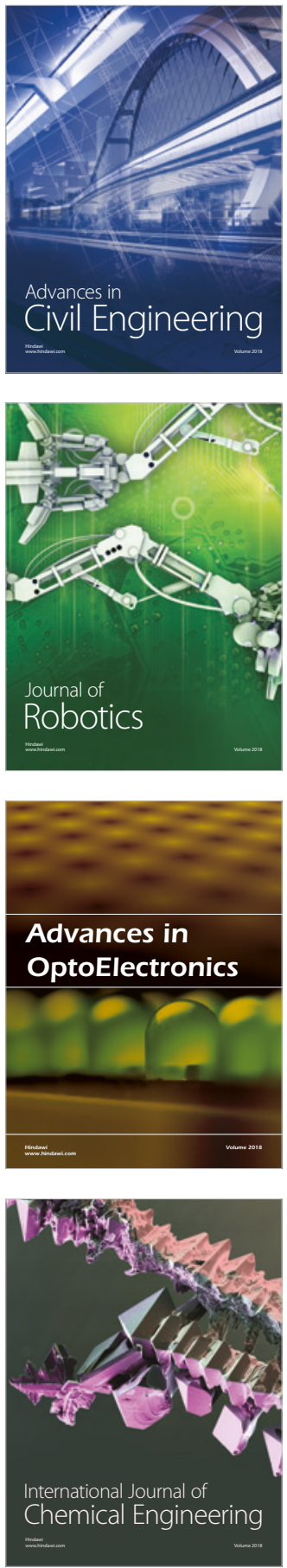

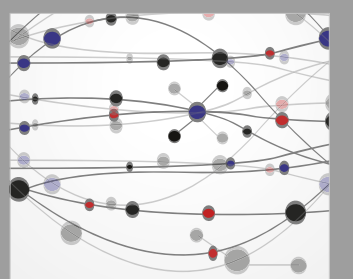

\section{Rotating \\ Machinery}

The Scientific World Journal

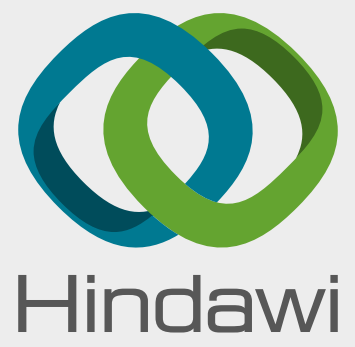

Submit your manuscripts at

www.hindawi.com
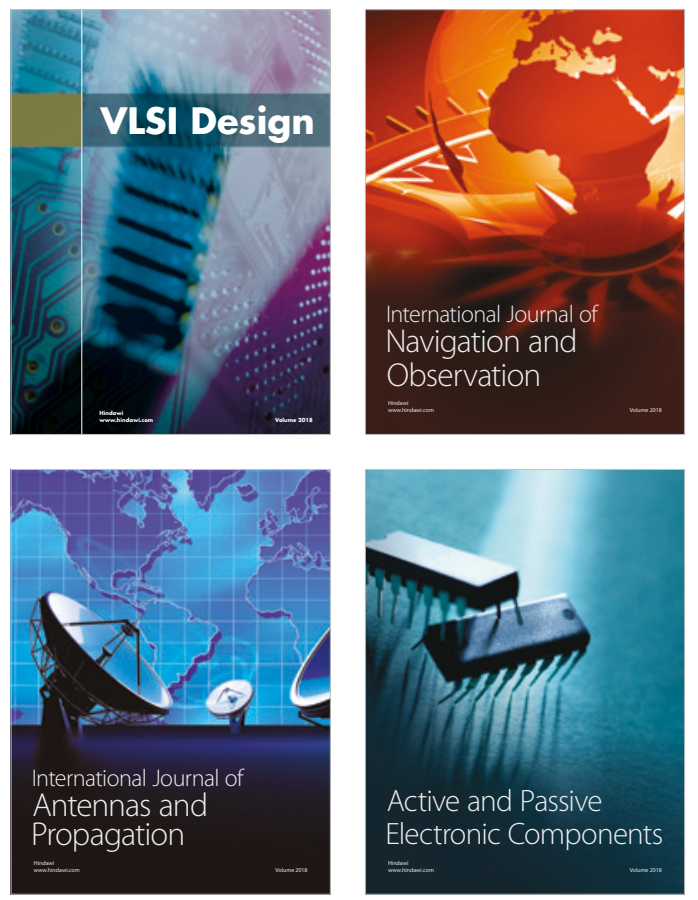
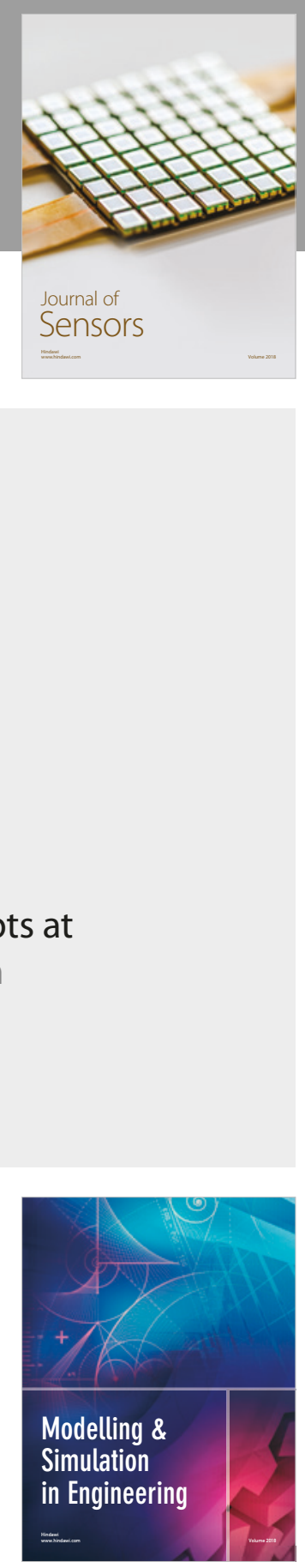

\section{Advances \\ Multimedia}
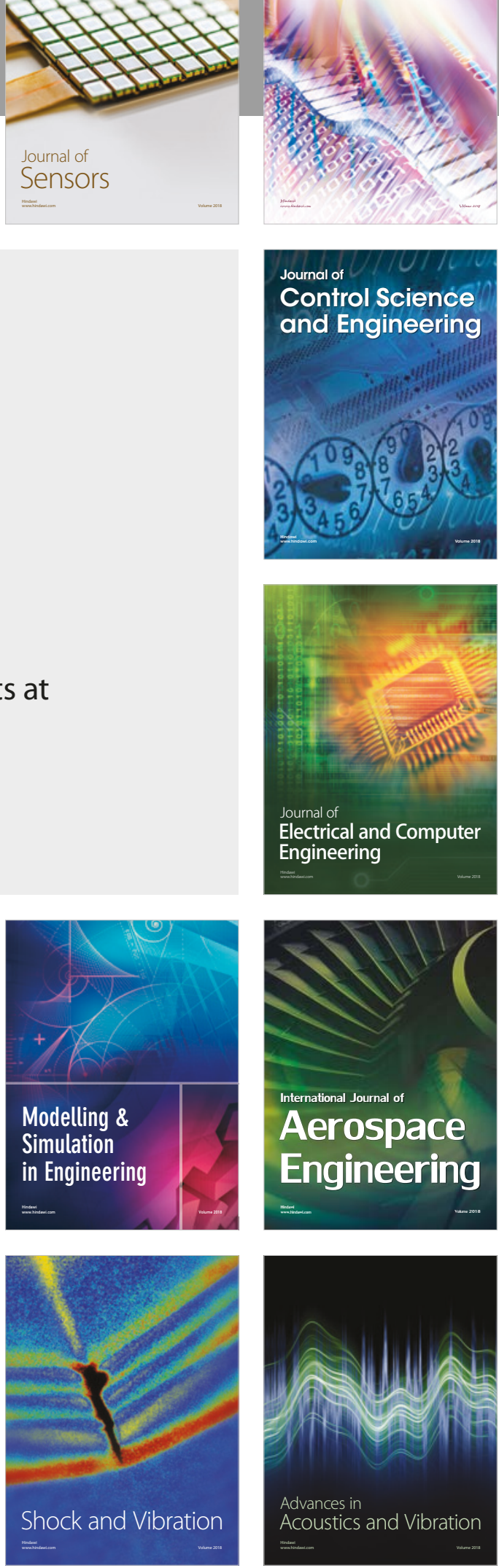